\title{
Utility of the Clinical Dementia Rating in Asian Populations
}

\author{
Wee Shiong Lim, MRCP, MMed; Mei Sian Chong, MRCP; and Suresh Sahadevan, FRCP
}

\begin{abstract}
Consistent with the worldwide demographic trend of population aging, dementia is expected to become a burgeoning public health problem in Asian populations. Thus, there is a pressing need for reliable and valid methods of dementia diagnosis and staging that are applicable in heterogeneous Asian populations. The Clinical Dementia Rating (CDR) is an informant-based global assessment scale with established reliability and validity that has been widely utilized as a severity-ranking scale in many studies of Asian populations. From a diagnostic standpoint, the CDR is congruent with the Diagnostic and Statistical Manual of Mental Disorders approach of dementia diagnosis. It exhibits excellent discriminatory ability in the very mild stages of dementia, a useful property that is germane to the surging interest in mild cognitive impairment and related concepts. Limitations of the CDR include its length of administration, reliance on clinical judgment and collateral source information, and relative insensitivity as a measure of change in interventional studies. Since the exercise of clinical judgment is inherent in scoring, CDR raters should be mindful of the influence of cultural factors on premorbid lifestyle, informant reliability and performance in certain CDR test items (especially those pertaining to the categories of judgment and problem solving, community, and home and hobbies). Thus, in future studies that involve the nascent use of the CDR in Asian populations, it is recommended that any transcultural adaptation of CDR items be described in detail and appropriate validation studies be carried out before adopting the CDR as a yardstick measure of assessment. The potential of adapted versions of the CDR in chronic care settings and advanced cases should be explored.An integrative approach, combining brief informant interview in conjunction with brief objective cognitive testing, could be a viable strategy for dementia screening in the clinical and research setting that warrants further evaluation in Asian populations.
\end{abstract}

Keywords: Asia; Classification; Cross-cultural comparison; Dementia; Diagnosis; Severity of illness index

$\mathrm{D}$ ementia accounts for $2 \%$ of years of life lived with disability, ahead of other chronic diseases, such as cerebrovascular disease and diabetes mellitus. ${ }^{1}$ With the worldwide demographic trend of population aging, Alzheimer's disease and other dementias will become an increasingly major public health problem among the elderly.

The public health implication of dementia is especially pertinent to Asian populations, which contribute almost half (46\%) of the worldwide 25 million demented persons (table 1). ${ }^{2}$ Existing disease burden can be estimated by cross sectional prevalence studies. The prevalence rate of dementia among persons aged 65 years and older has been variously reported

Reprint Requests: Wee Shiong Lim, MRCP, MMed, Department of Geriatric Medicine, Tan Tock Seng Hospital, I I Jalan Tan Tock Seng, Singapore 308433. Tel: 65-63577859, Fax: 65-6357837,Email:Wee_Shiong_Lim@ttsh.com.sg as $1.8 \%$ to $6.3 \%$ in Chinese, ${ }^{3-5} 3.3 \%$ to $8.0 \%$ in Japanese, 6,7 $1.4 \%$ to $4.0 \%$ in Indian, $8,98.2 \%$ to $10.8 \%$ in Korean ${ }^{10}$ and $2.5 \%$ to $7.7 \%$ in South-East Asian 11,12 populations. The wide range can be explained by the different sample characteristics, screening strategies and dementia ascertainment methods. The few prospective incidence studies reported rates ranging from 3.2 to 8.9 per 1000-person years among those aged 65 years and older, which is lower than in the United States or Europe. ${ }^{13}$ Going forward, dementia incidence and prevalence are expected to increase exponentially in Asian populations. ${ }^{2}$ In Japan, for example, the prevalence of dementia patients is expected to triple from 1 million in 1990 to 2.9 million by the year 2020.6
Revised:August 3, 2006

Accepted: October 19, 2006
DOI: 10.3121/cmr.2007.693 
Table 1. Worldwide occurrence of dementia in year $2000 .^{2}$

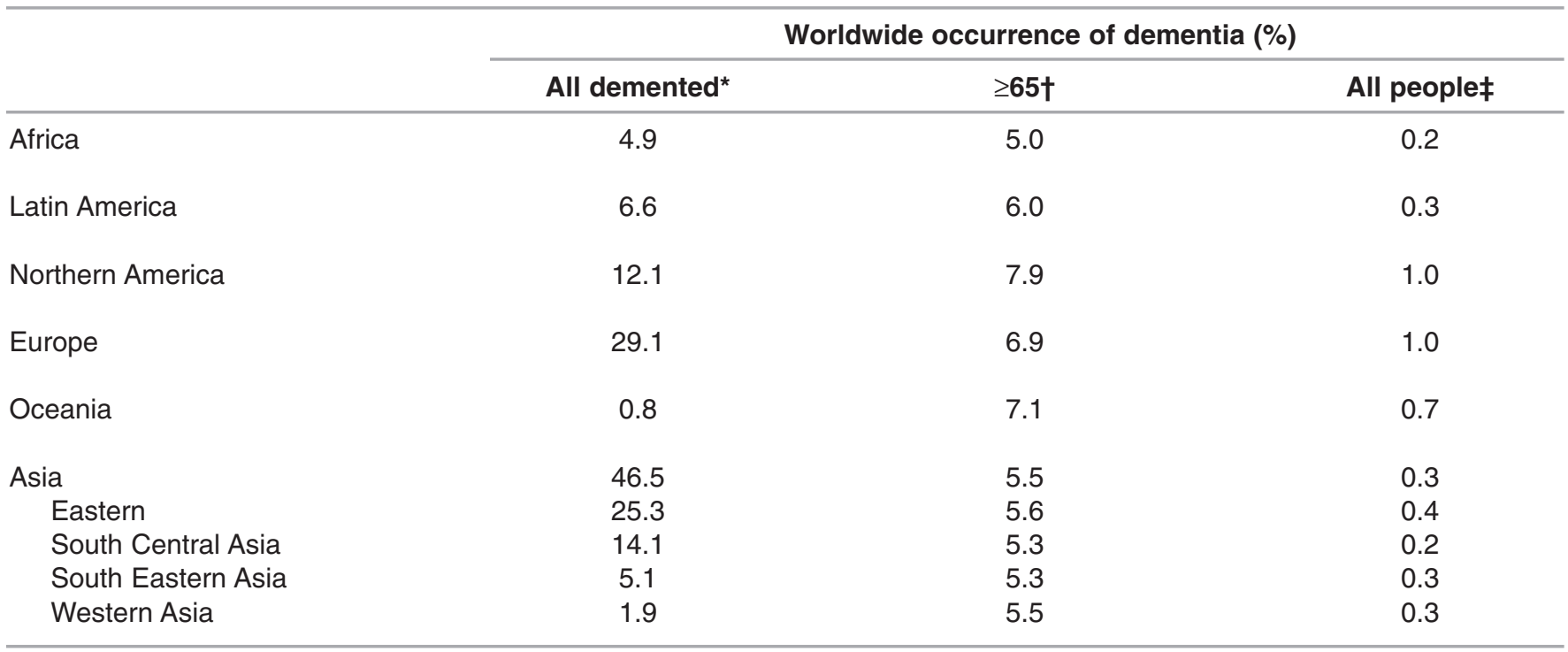

*Number of demented (all) as a proportion of all demented.

†Number of demented (65 years and older) as a proportion of all persons 65 years and older.

$\ddagger$ Number of demented (all) as a proportion of all people (demented and non-demented).

This defining demographic trend raises the clarion call for reliable and valid methods of dementia diagnosis and staging that are applicable to Asian populations. Challenges abound in the transcultural adaptation of dementia rating scales that are mainly derived from Caucasian populations. There is a high rate of illiteracy and lack of formal education among many of the elderly in Asian populations, especially in rural settings, such that any assessment tool emphasizing numeracy or literacy, even in their first or native language, may underestimate the cognitive abilities of a large proportion of Asian ethnic groups. ${ }^{14}$ Culturally-biased assessment methods that assume familiarity with a particular culture (usually Western) would also unfairly bias against culturally-diverse Asian populations with their own distinctive cultural characteristics. If not addressed, these factors could give rise to a well-documented tendency to over-classify some cases in minority ethnic groups with no disease as being impaired. ${ }^{15}$

In this regard, objective dementia rating scales that utilize mental status tests or more detailed neuropsychological assessment to establish the presence of cognitive impairment would be particularly vulnerable to many of the challenges outlined above. Ganguli and colleagues ${ }^{16}$ reported that the Mini-Mental State Examination (MMSE) required substantial revision or substitution of many items to arrive at a culturally and educationally fair assessment for use in northern India. In contrast, subjective (or global) assessment methods ascertain changes in behavior and everyday functioning brought about by cognitive decline in the context of an individual's past performance. Because the individual himself serves as the basis of comparison, global scales are less susceptible to floor and ceiling effects, or bias due to educational level or cultural differences. ${ }^{17}$ They provide a video perspective of longitudinal progression, as opposed to the cross-sectional snapshot afforded by a single result on objective testing. 18 Global measures can also be administered to informants of subjects who are cognitively untestable (such as sensory impairment, severe dementia or acute illness), including retrospective informant-based assessments in deceased individuals. ${ }^{19}$

The Clinical Dementia Rating (CDR) is a global clinical scale with established diagnostic and severity-ranking utility that has been widely employed in epidemiological studies, case series and clinical trials in an international context. ${ }^{20}$ It has also been adapted for routine clinical use in tertiary clinics ${ }^{21}$ and chronic care facilities. ${ }^{22}$ The CDR encompasses the strengths of the global approach outlined above. By evaluating cognitive performance with regards to the individual's accustomed functioning in everyday tasks, such that cognitive demands are imposed by one's natural environment within his own cultural and ethnic context, the CDR demonstrates good face validity and is generally more robust against the influence of cultural bias. ${ }^{23}$ In addition, it has good inter-rater reliability, ${ }^{24}$ concurrent validity as judged by psychometric 25 and neuropathologic correlation, ${ }^{26}$ and predictive validity as judged by longitudinal data. ${ }^{27}$ Although primarily developed for use in Alzheimer's disease, it has been employed for evaluation of non-Alzheimer dementias.

There have been increasingly more published studies of Asian populations that employ the CDR as a dementia ascertainment scale. In this review, we evaluate the evidence for its utility in Asian populations with regards to its validation and application as a global measure of dementia diagnosis, staging and change. We also highlight considerations pertinent to its use and further research in the Asian context. 
SEARCH STRATEGY AND SELECTION CRITERIA

A comprehensive, systematic MEDLINE search (1990 through July 15, 2006 with no language restrictions) was conducted using the terms "clinical dementia rating", "CDR", "Asia*", "Chinese", "Japan*”, "Korea*”, "India*”, "ethnic*”, "dementia" and "diagnosis", and their various combinations. The search was further supplemented by references from retrieved articles and through searches of the authors' own files for literature not covered in MEDLINE. For the purpose of this review, we did not exclude Western studies that also included minority Asian ethnic groups in the areas studied.

\section{THE CDR SCALE}

The CDR was developed at Washington University School of Medicine and first published in $1982 .{ }^{20}$ It was last revised in 1993.28 The CDR has established inter-rater reliability and can be administered by any trained personnel (usually a clinician or trained nurse). ${ }^{29,30}$ It employs a semi-structured interview with both the patient and a reliable informant (usually spouse or adult children) to rate performance in six domains: memory, orientation, judgment and problem solving, community affairs, home and hobbies, and personal care. Each domain is rated (independent of dysfunction caused by non-cognitive factors) according to one of five levels of impairment: $0=$ none, $0.5=$ questionable, $1=$ mild, $2=$ moderate, $3=$ severe.

The global CDR score is then assigned according to published rules to indicate the presence or absence of dementia, and when present, its severity. ${ }^{28}$ A CDR of 0 indicates no dementia. CDRs of $0.5,1,2$ and 3 indicate very mild, mild, moderate, and severe dementia, respectively. The CDR 0.5 category can be further divided into two diagnostic subcategories: (1) very mild or incipient dementia and (2) uncertain dementia, comprising individuals with cognitive impairment of insufficient degree to interfere with everyday functioning or possessing another potentially reversible condition (such as depression) that may account for the questionable impairment. ${ }^{31}$ In some studies, two additional stages are included for the most severe stages of dementia. ${ }^{32,33}$ Stage 4 describes profound dementia, in which persons have severe impairment in language or comprehension, inability to walk or eat without assistance, problems recognizing their family, and incontinence. Stage 5 refers to terminal dementia, in which persons require total care and are completely uncommunicative, bedridden, vegetative and incontinent. To provide a more quantitative measure of dementia severity, the ratings in each of the six categories can be totaled to yield the sum of boxes score (range: 0-18), which has been employed in clinical trials to track longitudinal progression. ${ }^{23}$

The utility of the CDR as a staging instrument is enhanced by two inherent characteristics: (1) property of hierarchy, whereby a global score of 3 represents a worse severity than 2 , which in turn is more severe than 1 , and so on across all patients and (2) property of concordance in the scores of its six domains, such that in a typical case of mild dementia of the Alzheimer type, most scores in the different domains would hover around 0.5 or 1.17 The presence of widely divergent scores (such as a mixture of $0 \mathrm{~s}, 2 \mathrm{~s}$ and $3 \mathrm{~s}$ ) would alert the clinician to the possibility of a non-Alzheimer cause of dementia.

\section{EVIDENCE OF CDR USE IN ASIAN POPULATIONS Validation Studies}

Many Asian studies assume the transcultural validity of the CDR and employ it as a gold standard of dementia assessment without local validation. We uncovered only two explicit Asian validation studies that established the reliability and validity of the CDR. ${ }^{21,34}$ One study in a multi-racial Asian population reported the convergent validity of the CDR when compared against clinical features, psychometric measures and the Diagnostic and Statistical Manual of Mental Disorders, $3^{\text {rd }}$ edition (DSM-III)-R criteria. The CDR showed good correlation with the Chinese MMSE $(r=0.73, p<0.01)$, the abbreviated mental test $(\mathrm{r}=0.73, \mathrm{p}<0.01)$ and neuropsychological tests of verbal memory $(\mathrm{r}=0.60-0.66$, $\mathrm{p}<0.01)$ and category fluency $(\mathrm{r}=0.62, \mathrm{p}<0.01) .{ }^{21}$ This underlined the transcultural feasibility of the CDR extending across the three studied races (Chinese, Malay and Indian) and suggested that there are no peculiar racial or language barriers to its use.

Predictive validity is supported by longitudinal data in two Japanese studies of the utility of CDR staging in describing predictive features of progression. ${ }^{35,36}$ Similar to data from the US and Europe, a recent Korean study reported that in subjects with mild cognitive impairment (MCI), the CDR sum of boxes score (especially when combined with word list recall) is a good predictor of progression to Alzheimer's disease. ${ }^{37}$ Further evidence of the validity of CDR staging can be indirectly accrued from other Asian neuropathological, ${ }^{38}$ genetic $^{39,40}$ and neuroimaging studies. . $^{6,41,42}$

\section{Dementia Diagnosis}

Because the CDR also defines non-demented aging $(\mathrm{CDR}=0)$, it additionally has, in practice, the diagnostic capacity of distinguishing no dementia from dementia even in its mildest stages (i.e., $\mathrm{CDR}=0.5$ ). ${ }^{17}$ The $\mathrm{CDR}$ has been demonstrated to be congruent with the DSM-IV approach of identifying dementia in a multi-racial Asian population. ${ }^{21}$ Both assess cognitive domains, while the functional domain (community, home and hobbies, and personal care) ratings of the CDR neatly enable the functional decline component of the DSM approach of dementia diagnosis to be systematically looked for.

The CDR has been employed as part of a multi-pronged strategy for the detection of dementia in Asian epidemiological studies in urban ${ }^{43-45}$ and rural settings (e.g., the Tajiri Project ${ }^{6}$ and the US-Indo study 8,13 ). Although primarily used as a staging instrument in these studies, the CDR complements the DSM criteria for the purpose of dementia diagnosis, and 
it has the added advantage of being able to evaluate both Alzheimer and non-Alzheimer forms of dementia. Most studies employ CDR $\geq 0.5$ as the cutoff for dementia, while others use CDR $\geq 1$ as the cutoff. ${ }^{39,40}$ While other informant-based measures such as the Informant Questionnaire on Cognitive Decline in the Elderly (IQCODE), ${ }^{12,46}$ Blessed Dementia Rating Scale, ${ }^{47}$ Community Screening Instrument for Dementia, ${ }^{48,49}$ Everyday Abilities Scale for India ${ }^{50}$ and the Instrumental Activities of Daily Living Scale for elderly people 51 have been used as screening instruments for dementia in Asian community studies, we did not identify similar studies for the CDR. One study reported how a more abbreviated format of CDR administration integrated into the DSM-IV approach of identifying dementia was adapted for use in a clinical service. ${ }^{21}$

\section{Measure of Severity}

This is the most widely used application of the CDR. Compared with the DSM-III-R staging criteria, the CDR demonstrated moderately good agreement $(k a p p a=0.58)$ and yielded comparatively better discriminatory ability in the milder stages of dementia. ${ }^{21}$ The utility of the 7 -stage CDR (inclusive of stages 4 and 5 for more severe stages of dementia) in Asian populations is supported by an American multi-ethnic community study of minority groups, including Asian Americans, that examined the stage-specific prevalence of behavioral symptoms extending across the spectrum of Alzheimer's disease severities. ${ }^{33}$

The utility of the CDR as a staging tool has been exploited in different ways. It has been employed in local validation studies of evaluative instruments, whether adapted from non-Asian populations (e.g., IQCODE, ${ }^{46}$ Community Screening Instrument for Dementia, ${ }^{48}$ Clock Drawing Test, ${ }^{52}$ Cognitive Abilities Screening Instrument,53,54 Rivermead Behavioural Memory Test, ${ }^{55}$ Alzheimer's Disease Assessment Scale Cognitive Subscale, ${ }^{56}$ Consortium to Establish a Registry for Alzheimer's Disease psychometric battery, ${ }^{57}$ Quality of Life-Alzheimer's Disease, ${ }^{58}$ Severe Impairment Battery, ${ }^{59}$ Executive Interview ${ }^{60}$ ) or de nouveau (e.g., Everyday Abilities Scale for India, ${ }^{50}$ Instrumental Activities of Daily Living Scale for elderly people, ${ }^{51}$ short memory questionnaire ${ }^{61}$ ). The dementia staging ability of the CDR allows patients of appropriate severity to be selected for intervention, ${ }^{44,62,63}$ histopathological, ${ }^{38}$ genetic ${ }^{39,40}$ and caregiving 58,64 studies in Asian populations. It also affords the examination of stage-dependent manifestations, including cognitive features, ${ }^{21,65,66}$ neuroimaging characteristics ${ }^{6,41,42}$ and behavioral issues. $33,67,68$

For instance, affective and anxiety disturbances tend to be prevalent in the mild-moderate stages of disease, whereas sleep disturbances, delusions and hallucinations are more common in the moderate-to-severe stages. ${ }^{33}$ Insofar as this and other studies in Asian populations reveal essentially similar findings to those in Caucasian populations, they support the transcultural cognitive and behavioral manifestation of dementia. $21,33,66$ This contrasts with the results of inter-ethnic studies that note the existence of cross-cultural differences in health-seeking behavior. Thus, Chow and colleagues ${ }^{69}$ reported that Chinese American dementia patients tended to present later compared with Caucasian Americans. Another study in an Asian population has shown, however, that it was the non-Chinese patients (Indians and Malays) who initially presented at more advanced stages compared with Chinese patients. ${ }^{21}$

A major strength of the CDR as a severity-staging instrument is its discriminatory ability even in the very mild or incipient stages of dementia. ${ }^{21}$ This could be attributable to the CDR's emphasis on cognitive functional decline relative to one's past performance, as opposed to an absolute definition of clear-cut cognitive impairment with loss of functional independence. The former would represent a more sensitive, and arguably more appropriate, threshold for detection of the subtle cognitive functional changes in the early stages of dementia. 70,71 Thus, the CDR 0.5 group is well placed for use in studies that seek to examine intermediate states between normal aging and dementia, such as MCI (table 2). ${ }^{72}$

Available studies in Asian populations add to the growing body of evidence in Caucasian populations that the CDR 0.5 group is similar to very mild Alzheimer's disease with regards to cognitive features, ${ }^{35,61,73}$ behavior, ${ }^{67}$ psychometric performance, $72,74,75$ and longitudinal progression, $, 35,37,76$ as well as structural and functional neuroimaging characteristics. ${ }^{41,42}$ Many of these studies utilize a more liberal definition of MCI that equates to CDR 0.5 , although some studies subclassify the CDR 0.5 group into MCI not demented (corresponding to CDR 0.5 uncertain dementia) and MCI incipient dementia (equivalent to CDR 0.5 incipient or very mild dementia). ${ }^{61,72}$ The prevalence of CDR 0.5 has been reported to be $30.2 \%$ among native Japanese in the Tajiri project and $29.5 \%$ among Japanese emigrants to Sao Paulo, Brazil. ${ }^{72,77}$ Prospective studies in Asian cohorts of CDR 0.5 subjects reveal rates of conversion to clinically-evident dementia which are comparable to the Mayo figures (6.5\%-19.9\% per year versus $10 \%-15 \%$ per year). $35,37,76$ About $40 \%$ in their cohort of community-dwelling incipient dementia subjects do not self-report memory complaints, and thus the Tajiri investigators suggest that the CDR 0.5 concept of MCI is more applicable to community residents compared with the Petersen amnestic MCI criteria which emphasizes subjective memory complaints. ${ }^{72}$

\section{Measure of Change}

Global measures of change to measure meaningful clinical change in anti-dementia drug trials are mandated by FDA guidelines. A critical consideration for this purpose is the sensitivity of these instruments in detecting the modest benefits of anti-dementia drugs. We did not identify any study of Asian populations that utilized the CDR primarily in this capacity. In this regard, the CDR has been comparatively less useful as compared to other global measurement scales, such as the Clinician's Interview-based Impression of Change-plus, ${ }^{78}$ Clinician's Global Impression of Change ${ }^{79,80}$ and IQCODE. ${ }^{79}$ 
Table 2. Selected studies of mild cognitive impairment (MCI) in Asian populations.

\begin{tabular}{|c|c|c|c|c|c|}
\hline Reference & Setting & Study population & $\mathrm{MCl}$ criteria & Key findings & Comments \\
\hline $\begin{array}{l}\text { Meguro } \\
2004^{72}\end{array}$ & $\begin{array}{l}\text { Rural: } \\
\text { community } \\
\text { dwelling } \\
\text { subjects } \\
\text { recruited from } \\
\text { Tajiri project } \\
\text { in Japan }\end{array}$ & $\begin{array}{l}\mathrm{N}=453 \\
\text { Mean age=74.8 } \\
\text { Baseline } \\
\text { MMSE=23.8 } \\
\text { Baseline } \\
\text { CASI=80.6 }\end{array}$ & $\begin{array}{l}\text { CDR } 0.5 \text { classified into } \\
3 \text { groups (UD, ID, } \\
\text { DAT) and compared } \\
\text { with } 3 \mathrm{MCl} \text { subtypes } \\
\text { (Petersen; Memory } \\
\text { complaint free; Short } \\
\text { term memory } 1.5 \mathrm{SD} \text { ) }\end{array}$ & $\begin{array}{l}\text { 1. Widely differing } \\
\text { prevalence rates } \\
\text { between CDR } 0.5 \\
(30.2 \%) \text { and } \mathrm{MCl} / \text { Petersen } \\
(4.9 \%) \text { groups } \\
\text { 2. Cognitive test performance } \\
\text { of CDR } 0.5 \text { subjects } \\
\text { intermediate between } \\
\text { healthy elderly and } \\
\text { demented groups }\end{array}$ & $\begin{array}{l}\text { 1. Reliability of } \\
\text { Japanese version of } \\
\text { CDR previously } \\
\text { established. } \\
\text { 2. CDR assessment } \\
\text { supplemented by nurse } \\
\text { visit to subjects' homes } \\
\text { to evaluate daily } \\
\text { activities }\end{array}$ \\
\hline
\end{tabular}

\begin{tabular}{|c|c|c|c|c|c|}
\hline $\begin{array}{l}\text { Lee } \\
2006^{37}\end{array}$ & $\begin{array}{l}\text { Urban: subjects } \\
\text { attending one } \\
\text { public health } \\
\text { center and two } \\
\text { memory clinics } \\
\text { in Korea }\end{array}$ & $\begin{array}{l}\mathrm{N}=106 \\
\text { Mean age }=73.1 \\
63 \% \text { female } \\
\text { Baseline CDR- } \\
\qquad \mathrm{SB}=1.2\end{array}$ & CDR 0.5 QD & $\begin{array}{l}\text { 1. At the end of } 3 \\
\text { years, } 8.3 \% \text { improved; } \\
72.7 \% \text { remained stable; } \\
\text { 19.4\% progressed to } A D \\
\text { 2. Addition of ApoE } \\
\text { genotype to CDR-SB or } \\
\text { word list recall did not } \\
\text { improve their predictive } \\
\text { ability of } A D \text { progression }\end{array}$ & $\begin{array}{l}\text { 1. Dropout rate }=32 \% \\
\text { 2. Investigators CDR } \\
\text { certified; inter-rater } \\
\text { agreement }=100 \% \\
\text { 3. CDR } 0.5 \mathrm{QD} \text { not } \\
\text { subdivided into UD } \\
\text { versus ID groups }\end{array}$ \\
\hline $\begin{array}{l}\text { Wang } \\
2006^{76}\end{array}$ & $\begin{array}{l}\text { Urban: community } \\
\text { dwelling subjects } \\
\text { recruited from } \\
\text { Veterans } \\
\text { General Hospital } \\
\text { study in Taiwan }\end{array}$ & $\begin{array}{l}\mathrm{MCl} \text { cohort } \\
\mathrm{N}=58 \\
\text { Mean age }=76.3 \\
26 \% \text { female } \\
\text { Baseline } \mathrm{CASI}=84.1 \\
\text { Baseline MMSE- } \\
\qquad \mathrm{CE}=25.9\end{array}$ & $\begin{array}{l}\text { Two definitions: } \\
\text { 1.CDR } 0.5 \mathrm{QD} \\
\text { 2.Petersen amnestic } \\
\mathrm{MCl} \text { criteria }\end{array}$ & $\begin{array}{l}\text { 1. Similar conversion } \\
\text { rates between CDR } 0.5 \\
\text { QD and amnestic } \mathrm{MCl} \\
\text { groups (19.9\% vs } 18.2 \% \\
\text { person-year) } \\
\text { 2. Smaller hippocampi } \\
\text { and lower cognitive } \\
\text { performance at baseline } \\
\text { predicted conversion } \\
\text { to } \mathrm{AD}\end{array}$ & $\begin{array}{l}\text { 1. Different duration of follow } \\
\text { up between CDR } 0.5 \mathrm{QD} \\
\text { (5 yr) and amnestic } \\
\mathrm{MCl}(3 \mathrm{yr}) \\
\text { 2. CDR } 0.5 \mathrm{QD} \text { not } \\
\text { subdivided into UD } \\
\text { versus ID groups }\end{array}$ \\
\hline $\begin{array}{l}\text { Meguro } \\
2004^{35}\end{array}$ & $\begin{array}{l}\text { Rural: community } \\
\text { dwelling subjects } \\
\text { recruited from } \\
\text { Tajiri project in } \\
\text { Japan }\end{array}$ & $\begin{array}{l}\mathrm{N}=55 \\
\text { Mean age }=74 \\
45.4 \% \text { female } \\
\text { Baseline } \mathrm{CASI}=78\end{array}$ & CDR 0.5 QD & $\begin{array}{l}\text { 1. At end of } 3 \mathrm{yr}, 34.5 \% \\
\text { progressed to } A D \\
\text { 2. Informant report } \\
\text { rather than self-report } \\
\text { of memory decline } \\
\text { differentiated CDR } 0 \\
\text { from CDR } 0.5 \text { group }\end{array}$ & $\begin{array}{l}\text { 1. Reliability of Japanese } \\
\text { version of CDR } \\
\text { previously established } \\
\text { 2. CDR assessment } \\
\text { supplemented by nurse } \\
\text { by nurse visit to subjects' } \\
\text { homes to evaluate } \\
\text { daily activities } \\
\text { 3. CDR } 0.5 \text { QD not } \\
\text { subdivided into UD versus } \\
\text { ID groups }\end{array}$ \\
\hline $\begin{array}{l}\text { Lam } \\
2006^{75}\end{array}$ & $\begin{array}{l}\text { Urban: ambulatory } \\
\text { Chinese subjects } \\
\text { recruited from } \\
\text { community social } \\
\text { centers and } \\
\text { residential hostels } \\
\text { in Hong Kong }\end{array}$ & $\begin{array}{l}\mathrm{N}=150 \\
\text { Mean age }=78.9 \\
\text { Baseline } \\
\text { CMMSE=23.3 }\end{array}$ & CDR 0.5 QD & $\begin{array}{l}\text { 1. CDR } 0.5 \text { had CVFT } \\
\text { scores intermediate } \\
\text { between normal } \\
\text { controls and AD } \\
\text { 2. Different predictors of } \\
\text { CVFT scores in the } \\
\text { normal control and CDR } \\
0.5 \text { groups }\end{array}$ & $\begin{array}{l}\text { 1. Authors further divided } \\
\text { CDR } 0.5 \text { group into } 2 \\
\text { subgroups based on } \\
\text { number of domains } \\
\text { of CDR affected } \\
\text { 2. Single trained geriatric } \\
\text { psychiatrist scored CDR }\end{array}$ \\
\hline
\end{tabular}

AD, Alzheimer disease; ApoE, Apolipoprotein E; CASI, Cognitive Abilities Screening Instrument; CDR, Clinical Dementia Rating Scale; CDR-SB, CDR Sum of Boxes score; CMMSE, Chinese Mini-Mental State Examination; CVFT, Category Verbal Fluency Test; DAT, Dementia of Alzheimer type; ID, Incipient dementia; MMSE, Mini-Mental State Examination; MMSE-CE, CASI-estimated Mini-Mental State Examination; QD, Questionable dementia; UD, Uncertain dementia. 


\section{COMMENTARY}

\section{Utility of CDR in Asian Populations}

The CDR is a comprehensive, reliable and valid informant-based global assessment method that has been successfully employed in dementia studies of Asian populations. By assessing change from the individual's prior level of functioning and tapping on one's accustomed functioning in daily tasks as the basis of cognitive assessment, the CDR demonstrates cross-cultural portability that is applicable across different educational levels and social settings. The semi-structured format permits flexibility in adapting the informant-based assessment to be more relevant and appropriate to the local culture and social setting. The CDR has also contributed to our understanding of incipient or very mild stages of dementia, such as MCI in Asian populations (table 2).

\section{Specific Considerations Regarding the Use of CDR in Asian Populations}

Reliance on Clinical Judgment

One major criticism of the CDR is the reliance on clinical judgment for scoring. Nonetheless, the CDR displayed good inter-rater reliability in two Asian validation studies and is highly correlated with brief objective measures (such as MMSE and abbreviated mental test) and more detailed psychometric testing. ${ }^{21,34}$ Moreover, the exercise of clinical judgment to critically appraise the informant account is inherent in all global scales and is essential for a panoramic overview of cognitive and functional abilities. The challenge lies in attuning definitions of impairment to consider prevailing societal norms and perception of normality, ${ }^{21}$ so that an accurate CDR rating that is truly reflective of the gestalt of the individual under evaluation can be derived. The CDR rater needs to be mindful of the tendency in many traditional Asian societies to downplay cognitive symptoms out of pious respect or low expectations of the elderly, or the perception of forgetfulness as a reflection of normal aging. ${ }^{13}$ One strategy is to corroborate collaborating information with direct observation of the performance of the individual in their everyday activities. ${ }^{35,72}$

\section{Reliance on Collateral Source Information}

A valid CDR assessment is predicated on the availability of reliable collateral sources who can provide information about a person's current level of cognitive functioning relative to previous levels. ${ }^{17}$ In many Asian societies, the elderly are venerated family members living together with their children and grandchildren in extended multi-tier families, and informal caregiving of disabled family members (including those with dementia) is socially mandated. ${ }^{81}$ Thus, a ready source of collateral information is often available in the form of spouse, adult children, relatives (not uncommonly, a daughter-in-law is tasked with the caregiving role), or in some instances, domestic helpers employed to look after the infirmed elderly. While the lack of a reliable informant who can provide a longitudinal perspective of change has not yet been a major issue in most published studies of Asian populations, this could change as Asian societies evolve and the younger generation becomes increasingly more disinclined to stay in traditional multi-tier families. Additionally, more research is required to determine if there are informant characteristics that can help predict the accuracy of collateral source reports. Available evidence from studies of Caucasian populations suggests that factors such as type of relationship to the patient, frequency of contact, whether they are living together, age, education, informant's mental health and quality of the informant-patient relationship can affect the quality of collateral source information. ${ }^{82}$

\section{Influence of Culture on CDR Scoring}

It is important to appreciate that Asian populations are vastly heterogeneous with regard to language, culture, religious beliefs, gender roles, socioeconomic status and social setting (urban versus rural). Many of the studies of Asian populations assume the transcultural validity of the CDR and do not provide details regarding how specific information related to the individual's cognitive and functional abilities required to score each CDR category is elicited. Notwithstanding the overall cross-cultural portability of the CDR as compared with many of the objective assessment tools, it is important to recognize that the CDR is not entirely free of cultural bias.

Certain items in the CDR can be deemed to be relatively ubiquitous and non-culturally-biased, for example, those that refer to the categories of memory (such as meal preparation, taking responsibility for one's medication, recalling conversations, remembering where things are usually kept $)^{45}$ and orientation. In contrast, other functional items that need to be queried in the CDR, especially those pertaining to judgment and problem solving, community, and home and hobbies categories, may be contextually dependent on the local culture (crossword puzzles and board games are uncommon hobbies in many Asian societies, whereas the packing of red packets is useful for screening of executive function among Chinese societies practicing this custom), social setting (looking after farm animals would apply to rural rather than urban settings), ${ }^{50}$ literacy (tasks such as telephone use and writing of checks), ${ }^{50}$ and gender roles (meal preparation is more applicable to the female gender in certain Asian societies). ${ }^{50,83}$

Similarly, evaluating calculation ability under the judgment and problem solving category would require adaptation to the local culture and social setting. For instance, even the seemingly straightforward serial sevens item on the MMSE may be considered too conceptually abstract for certain cultures. One possibility is to employ culture-free tests, such as the ability to calculate using the local currency, although there may still be concerns about item equivalence in terms of difficulty level. For example, in one study ${ }^{73}$ the question "how many 5 cents are in 1.35 local dollars?" was asked. It is debatable if the question would be of comparable difficulty to "how many quarters are there in USD 6.75?", even though the answer to both questions is 27. Cultural differences in the 
performance of certain CDR test items cannot be discounted. It is the authors' experience that there is disproportionately greater difficulty in conceptualization of similarities among the Chinese. Corroborating evidence comes from the validation study of the Chinese Frontal Assessment Battery, where it was reported that normal controls consistently scored lower on the similarities item (mean=0.71) compared with the other five items, including the differences item (range of means: $2.5-3.0) .84$

Not uncommonly, the protectiveness towards the elderly seen in many conservative Asian families, often encourages a longstanding restricted and homebound lifestyle with little variability in their daily routines and where most tasks are taken over by their families. 5,13 In such circumstances, the utility of testing recent autobiographical memory (which involves the recall of recent events in the last 1 week and 1 month) would be limited. Likewise, the ability to elicit discernible changes from a pre-morbidly low level of functioning can be extremely challenging, especially in the categories of community affairs, and home and hobbies.21 The rating of impairment is then not easily made when such individuals present in the early stages of dementia.

Taken together, this emphasizes the need for future studies that employ the CDR to describe the transcultural adaptation and considerations unique to the local culture and study setting. In transcultural Asian studies that involve the nascent use of the CDR, it is recommended that appropriate validation studies be done first to establish the validity and reliability of the CDR.

\section{Limitations of CDR in Specific Scenarios}

This review highlighted issues in Asian populations that are akin to those in Caucasian populations. One such analogous situation is the assessment of nursing home residents, where practical difficulties exist in establishing a meaningful degree of cognition-related functional decline. This is an area that is expected to feature more prominently in the coming years with the increasing industrialization and erosion of the nuclear family unit in many Asian countries. The potential of a chronic care facility adaptation of the CDR scale could be explored for use in the Asian context. ${ }^{22}$

The standard 5-stage CDR does not cater to the measurement of meaningful changes in the more advanced stages of dementia, a need that has become more salient with the arrival of memantine. Future studies should examine the reliability and validity of the 7-stage CDR scale 32,33 in advanced dementia patients. Moreover, the CDR is comparatively less sensitive for gauging the modest benefits of anti-dementia intervention as compared with other global measures of change, such as Clinician's Global Impression of Change or Clinician's Interview-based Impression of Change-plus, and has not found wide usage thus far in intervention studies. ${ }^{78-80}$
The CDR in Widespread Clinical Use

In its current format, the CDR is fairly resource intensive and probably more applicable to research settings. It requires the availability of trained raters, has a fairly complicated scoring system and takes longer than an average office visit permits (clinical interview of the patient and informant together average approximately 60-90 minutes). While the CDR has been successfully incorporated into the workflow of a tertiary referral clinic in an Asian setting, ${ }^{21}$ it still remains too cumbersome for general clinical use or as a screening tool in population studies. More research is required to develop and test the applicability of brief informant-based measures comprising high-yield clinical variables that are derived from the original battery of evaluative questions in the CDR interview. An example is the 8-item AD8 informant interview developed by Washington University. The AD8 takes less than 3 minutes to complete, can be administered by clerical staff and demonstrates good discriminatory ability between non-demented (CDR 0 ) and very mild dementia (CDR 0.5 ) groups (area under receiver operator curve $=0.834$, sensitivity $74 \%$, specificity $86 \%) .85$

Ultimately, the optimal performance in Asian settings is likely to be achieved by an integrative approach that combines informant interview with objective cognitive testing, which is the common, albeit impromptu, practice in many clinical settings. This premise is supported by studies of Asians in urbanized ${ }^{48,49}$ and rura ${ }^{49,50}$ settings, as well as a community healthcare screening program to detect community-dwelling older persons in earlier stages of cognitive decline, ${ }^{44}$ where a combined approach is superior to either measure alone. Collaborative information from objective testing can also facilitate assessment in situations where a reliable informant is absent or lacking. The combination of brief objective testing in conjunction with a brief informant interview could prove to be a viable strategy for dementia screening in clinical trials, community surveys and epidemiologic studies. 85,86

\section{CONCLUSION}

The CDR is a versatile global assessment scale with established reliability and validity that has found widespread use as a severity-rating instrument in many studies of Asian populations. From a diagnostic standpoint, its excellent discriminatory ability in the very mild stages of dementia paves the way for its use as a key staging tool in emerging studies examining MCI and related concepts.

Drawbacks to the CDR include its length of administration, reliance on clinical judgment and availability of a reliable informant, and relative insensitivity as a global measure of change. The scoring of certain CDR domains, in particular the categories of judgment and problem solving, community, and home and hobbies, may also be subject to the influence of cultural factors and social settings. Thus, despite the established reliability and validity of the CDR in some Asian populations, ${ }^{21,34,37}$ its cross-cultural portability should be not assumed across all Asian populations. In transcultural Asian studies involving the nascent use of the CDR, it is 
recommended that transcultural adaptation of CDR items be described and the validity and reliability of the CDR be established first before its adoption as a gold standard for assessment. Future research in Asian populations should examine the role of the CDR in chronic care settings and advanced cases, informant characteristics that are predictive of collateral source accuracy, and the derivation of a brief informant interview that has clinical and research utility as a dementia screening tool.

\section{REFERENCES}

1. World Health Organization (WHO). Burden of mental and behavioural disorders. In: The world health report 2001: mental health: new understanding, new hope. Geneva, Switzerland: World Health Organization;2001. 19-45.

2. Wimo A, Winblad B, Aguero-Torres H, von Strauss E. The magnitude of dementia occurrence in the world. Alzheimer Dis Assoc Disord 2003;17:63-67.

3. Zhang MY, Katzman R, Salmon D, Jin H, Cai GJ, Wang ZY, Qu GY, Grant I, Yu E, Levy P, Klauber MR, Liu W. The prevalence of dementia and Alzheimer's disease in Shanghai, China: impact of age, gender, and education. Ann Neurol 1990;27:428-437.

4. Li G, Shen YC, Chen CH, Zhao YW, Li SR, Lu M. An epidemiological survey of age-related dementia in an urban area of Beijing. Acta Psychiatr Scand 1989;79:557-563.

5. Liu HC, Lin KN, Teng EL, Wang SJ, Fuh JL, Guo NW, Chou P, $\mathrm{Hu} \mathrm{HH}$, Chiang BN. Prevalence and subtypes of dementia in Taiwan: a community survey of 5297 individuals. J Am Geriatr Soc 1995;43:144-149.

6. Ishii H, Meguro K, Ishizaki J, Shimada M, Yamaguchi S, Sano I, Ambo H, Ohtake H, Shimada Y, Someya K, Sato M, Shibuya Y, Kato M, Sekita Y, Yamadori A. Prevalence of senile dementia in a rural community in Japan: the Tajiri project. Arch Gerontol Geriatr 1999;29:249-265.

7. Kiyohara Y, Yoshitake T, Kato I, Ohmura T, Kawano H, Ueda K, Fujishima M. Changing patterns in the prevalence of dementia in a Japanese community: the Hisayama study. Gerontology 1994;40:29-35.

8. Chandra V, Ganguli M, Pandav R, Johnston J, Belle S, DeKosky ST. Prevalence of Alzheimer's disease and other dementias in rural India: the Indo-US study. Neurology 1998;51:1000-1008.

9. de Silva HA, Gunatilake SB, Smith AD. Prevalence of dementia in a semi-urban population in Sri Lanka: report from a regional survey. Int J Geriatr Psychiatry 2003;18:711-715.

10. Lee DY, Lee JH, Ju YS, Lee KU, Kim KW, Jhoo JH, Yoon JC, Ha J, Woo JI. The prevalence of dementia in older people in an urban population of Korea: the Seoul study. J Am Geriatr Soc 2002;50:1233-1239.

11. Jitapunkul S, Kunanusont C, Phoolcharoen W, Suriyawongpaisal P. Prevalence estimation of dementia among Thai elderly: a national survey. J Med Assoc Thai 2001;84:461-467.

12. Lim HJ, Lim JP, Anthony P, Yeo DH, Sahadevan S. Prevalence of cognitive impairment amongst Singapore's elderly Chinese: a community-based study using the ECAQ and the IQCODE. Int J Geriatr Psychiatry 2003;18:142-148.

13. Chandra V, Pandav R, Dodge HH, Johnston JM, Belle SH, DeKosky ST, Ganguli M. Incidence of Alzheimer's disease in a rural community in India: the Indo-US study. Neurology 2001;57:985-989.

14. Parker C, Philp I. Screening for cognitive impairment among older people in black and minority ethnic groups. Age Ageing 2004;33:447-452.
15. Bohnstedt M, Fox PJ, Kohatsu ND. Correlates of Mini-Mental Status Examination scores among elderly demented patients: the influence of race-ethnicity. J Clin Epidemiol 1994;47:1381-1387.

16. Ganguli M, Ratcliff G, Chandra V, Sharma S, Gilby J, Pandav R, Belle S, Ryan C, Baker C, Seaberg E, Dekosky S. A Hindi version of the MMSE: the development of a cognitive screening instrument for a largely illiterate rural elderly population in India. Int J Geriatr Psychiatry 1995;10:367-377.

17. Sahadevan S, Rockwood K, Morris JC. Global assessment measures in dementia. In: Gauthier S, ed. Clinical Diagnosis and Management of Alzheimer's disease. $2^{\text {nd }} \mathrm{ed}$. London: Martin Dunitz; 1999:167-177.

18. Jorm AF, Christensen H, Henderson AS, Jacomb PA, Korten AE, Mackinnon A. Informant ratings of cognitive decline of elderly people: relationship to longitudinal change on cognitive tests. Age Ageing 1996;25:125-129.

19. Davis PB, White H, Price JL, McKeel D, Robins LN. Retrospective postmortem dementia assessment. Validation of a new clinical interview to assist neuropathologic study. Arch Neurol 1991;48:613-617.

20. Hughes CP, Berg L, Danziger WL, Coben LA, Martin RL. A new clinical scale for the staging of dementia. Br J Psychiatry 1982;140:566-572.

21. Lim WS, Chin JJ, Lam CK, Lim PP, Sahadevan S. Clinical dementia rating: experience of a multi-racial Asian population. Alzheimer Dis Assoc Disord 2005;19:135-142.

22. Marin DB, Flynn S, Mare M, Lantz M, Hsu MA, Laurans M, Paredes M, Shreve T, Zaklad GR, Mohs RC. Reliability and validity of a chronic care facility adaptation of the Clinical Dementia Rating scale. Int J Geriatr Psychiatry 2001;16:745-750.

23. Morris JC, Berg L, Cohen LA, Rubin EH, Deuel R, Wittenborn R, Coats M, Leon S, Norton J. In: Bergener M, Finkel SI, eds. Treating Alzheimer's and other dementias: clinical application of recent research advances. New York, NY: Springer Publishing;1995: 338-346.

24. Burke WJ, Miller JP, Rubin EH, Morris JC, Coben LA, Duchek J, Wittels IG, Berg L. Reliability of the Washington University Clinical Dementia Rating. Arch Neurol 1988;45:31-32.

25. Welsh KA, Butters N, Hughes JP, Mohs RC, Heyman A. Detection and staging of dementia in Alzheimer's disease. Use of the neuropsychological measures developed for the Consortium to Establish a Registry for Alzheimer's Disease. Arch Neurol 1992;49:448-452.

26. Berg L, McKeel DW Jr, Miller JP, Storandt M, Rubin EH, Morris JC, Baty J, Coats M, Norton J, Goate AM, Price JL, Gearing M, Mirra SS, Saunders AM. Clinicopathologic studies in cognitively healthy aging and Alzheimer's disease: relation of histologic markers to dementia severity, age, sex, and apolipoprotein E genotype. Arch Neurol 1998;55:326-335.

27. Berg L, Danziger WL, Storandt M, Coben LA, Gado M, Hughes CP, Knesevich JW, Botwinick J. Predictive features in mild senile dementia of the Alzheimer type. Neurology 1984;34:563-569.

28. Morris JC. The Clinical Dementia Rating (CDR): current version and scoring rules. Neurology 1993;43:2412-2414.

29. McCulla MM, Coats M, Van Fleet N, Duchek J, Grant E, Morris JC. Reliability of clinical nurse specialists in the staging of dementia. Arch Neurol 1989;46:1210-1211.

30. Morris JC, Ernesto C, Schafer K, Coats M, Leon S, Sano M, Thal LJ, Woodbury P. Clinical dementia rating training and reliability in multicenter studies: the Alzheimer's Disease Cooperative Study experience. Neurology 1997;48:1508-1510.

31. Villareal DT, Grant E, Miller JP, Storandt M, McKeel DW, Morris JC. Clinical outcomes of possible versus probable Alzheimer's disease. Neurology 2003;61:661-667. 
32. Heyman A, Wilkinson WE, Hurwitz BJ, Helms MJ, Haynes CS, Utley CM, Gwyther LP. Early-onset Alzheimer's disease: clinical predictors of institutionalization and death. Neurology 1987;37:980-984.

33. Chen JC, Borson S, Scanlan JM. Stage-specific prevalence of behavioral symptoms in Alzheimer's disease in a multi-ethnic community sample. Am J Geriatr Psychiatry 2000;8:123-133.

34. Otoyama W, Niina R, Homma A, et al. Inter-rater reliability of the Japanese version of CDR. Jpn J Geriatr Psyhciatry 2000;11:521-527.

35. Meguro K, Shimada M, Yamaguchi S, Sano I, Inagaki H, Matsushita M, Sekita Y, Mori E. Neuropsychosocial features of very mild Alzheimer's disease (CDR 0.5) and progression to dementia in a community: the Tajiri project. J Geriatr Psychiatry Neurol 2004;17:183-189.

36. Sauvaget C, Yamada M, Fujiwara S, Sasaki H, Mimori Y. Dementia as a predictor of functional disability: a four-year follow-up study. Gerontology 2002;48:226-233.

37. Lee DY, Youn JC, Choo IH, Kim KW, Jhoo JH, Pak YS, Suh KW, Woo JI. Combination of clinical and neuropsychologic information as a better predictor of the progression to Alzheimer disease in questionable dementia individuals. Am J Geriatr Psychiatry 2006;14:130-138.

38. Saito Y, Ruberu NN, Sawabe M, Arai T, Tanaka N, Kakuta Y, Yamanouchi H, Murayama S. Staging of argyrophilic grains: an age-associated tauopathy. J Neuropathol Exp Neurol 2004;63:911-918.

39. Chandak GR, Sridevi MU, Vas CJ, Panikker DM, Singh L. Apolipoprotein E and presenilin-1 allelic variation and Alzheimer's disease in India. Hum Biol 2002;74:683-693.

40. Ganguli M, Chandra V, Kamboh MI, Johnston JM, Dodge HH, Thelma BK, Juyal RC, Pandav R, Belle SH, DeKosky ST. Apolipoprotein E polymorphism and Alzheimer disease: The Indo-US Cross-National Dementia Study. Arch Neurol 2000;57:824-830.

41. Ishii K, Mori T, Hirono N, Mori E. Glucose metabolic dysfunction in subjects with a clinical dementia rating of 0.5 . J Neurol Sci 2003;215:71-74.

42. Ishii H, Meguro K, Yamaguchi S, Hirayama K, Tabuchi M, Mori E, Yamadori A. Different MRI findings for normal elderly and very mild Alzheimer's disease in a community: implications for clinical practice the Tajiri Project. Arch Gerontol Geriatr 2006;42:59-71.

43. Vas CJ, Pinto C, Panikker D, Noronha S, Deshpande N, Kulkarni L, Sachdeva S. Prevalence of dementia in an urban Indian population. Int Psychogeriatr 2001;13:439-450.

44. Fujiwara Y, Amano H, Mori S, Watanabe S, Kumagai S, Yoshida Y, Kim J, Takabayashi K, Yoshida H, Ishihara M, Eguchi F, Fuse S, Morita M, Nagai H, Shinkai S. Toward constructing a system for detecting and coping with senile dementia in early stages among community-dwelling older people. Nippon Koshu Eisei Zasshi 2003;50:739-748.

45. Graves AB, Larson EB, Edland SD, Bowen JD, McCormick WC, McCurry SM, Rice MM, Wenzlow A, Uomoto JM. Prevalence of dementia and its subtypes in the Japanese American population of King County, Washington state. The Kame Project. Am J Epidemiol 1996;144:760-771.

46. Fuh JL, Teng EL, Lin KN, Larson EB, Wang SJ, Liu CY, Chou P, Kuo BI, Liu HC. The Informant Questionnaire on Cognitive Decline in the Elderly (IQCODE) as a screening tool for dementia for a predominantly illiterate Chinese population. Neurology 1995;45:92-96.

47. Liu CK, Lin RT, Chen YF, Tai CT, Yen YY, Howng SL. Prevalence of dementia in an urban area in taiwan. J Formos Med Assoc 1996;95:762-768.

48. Liu SI, Prince M, Chiu MJ, Chen TF, Sun YW, Yip PK. Validity and reliability of a Taiwan Chinese version of the community screening instrument for dementia. Am J Geriatr Psychiatry 2005;13:581-588.
49. Prince M, Acosta D, Chiu H, Scazufca M, Varghese M; 10/66 Dementia Research Group. Dementia diagnosis in developing countries: a cross-cultural validation study. Lancet 2003;361:909-917.

50. Pandav R, Fillenbaum G, Ratcliff G, Dodge H, Ganguli M. Sensitivity and specificity of cognitive and functional screening instruments for dementia: the Indo-U.S. Dementia Epidemiology Study. J Am Geriatr Soc 2002;50:554-561.

51. Mathuranath PS, George A, Cherian PJ, Mathew R, Sarma PS. Instrumental activities of daily living scale for dementia screening in elderly people. Int Psychogeriatr 2005; 17:461-474.

52. Lin KN, Wang PN, Chen C, Chiu YH, Kuo CC, Chuang YY, Liu HC. The three-item clock-drawing test: a simplified screening test for Alzheimer's disease. Eur Neurol 2003;49:53-58.

53. Lin KN, Wang PN, Liu CY, Chen WT, Lee YC, Liu HC. Cutoff scores of the cognitive abilities screening instrument, Chinese version in screening of dementia. Dement Geriatr Cogn Disord 2002;14:176-182.

54. Liu HC, Teng EL, Lin KN, Chuang YY, Wang PN, Fuh JL, Liu CY. Performance on the cognitive abilities screening instrument at different stages of Alzheimer's disease. Dement Geriatr Cogn Disord 2002;13:244-248.

55. Matsuda A, Kazui H, Hirono N, Mori E. Validity of the Japanese version of Rivermead Behavioural Memory Test for evaluation of everyday memory function in patients with mild Alzheimer's disease. No To Shinkei 2002;54:673-678.

56. Chu LW, Chiu KC, Hui SL, Yu GK, Tsui WJ, Lee PW. The reliability and validity of the Alzheimer's Disease Assessment Scale Cognitive Subscale (ADAS-Cog) among the elderly Chinese in Hong Kong. Ann Acad Med Singapore 2000;29:474-485

57. Sahadevan S, Lim JP, Tan NJ, Chan SP. Psychometric identification of early Alzheimer disease in an elderly Chinese population with differing educational levels. Alzheimer Dis Assoc Disord 2002;16:65-72.

58. Fuh JL, Wang SJ. Assessing quality of life in Taiwanese patients with Alzheimer's disease. Int J Geriatr Psychiatry 2006;21:103-107.

59. Ahn IS, Kim JH, Ku HM, Saxton J, Kim DK. Reliability and validity of the severe impairment battery (SIB) in Korean dementia patients. J Korean Med Sci 2006;21:506-517.

60. Chan SM, Chiu FK, Lam CW. Correlational study of the Chinese version of the executive interview (C-EXIT25) to other cognitive measures in a psychogeriatric population in Hong Kong Chinese. Int J Geriatr Psychiatry 2006;21:535-541.

61. Lam LC, Lui VW, Tam CW, Chiu HF. Subjective memory complaints in Chinese subjects with mild cognitive impairment and early Alzheimer's disease. Int J Geriatr Psychiatry 2005;20:876-882.

62. Fukui T, Taguchi S. Do vascular lesions and related risk factors influence responsiveness to donepezil chloride in patients with Alzheimer's disease? Dement Geriatr Cogn Disord 2005;20:15-24

63. Mizuno S, Kameda A, Inagaki T, Horiguchi J. Effects of donepezil on Alzheimer's disease: the relationship between cognitive function and rapid eye movement sleep. Psychiatry Clin Neurosci 2004;58:660-665.

64. Takahashi M, Tanaka K, Miyaoka H. Depression and associated factors of informal caregivers versus professional caregivers of demented patients. Psychiatry Clin Neurosci 2005;59:473-480.

65. Fujiwara Y, Amano H, Takabayashi K, Kumagai S, Yoshida Y, Yoshida H, Mori S, Watanabe S, Morita M, Nagai H, Shinkai $\mathrm{S}$. Factors related to discrepancy in evaluation on functional capacity between reports by community-dwelling older people with cognitive decline and their family members. Nippon Ronen Igakkai Zasshi 2003;40:487-496. 
66. Mok EH, Lam LC, Chiu HF. Category verbal fluency test performance in chinese elderly with Alzheimer's disease. Dement Geriatr Cogn Disord 2004;18:120-124.

67. Chiu PY, Chung CL. Delusions in patients with very mild, mild and moderate Alzheimer's disease. Acta Neurol Taiwan 2006;15:21-25.

68. Fuh JL, Wang SJ. Assessing quality of life in Taiwanese patients with Alzheimer's disease. Int J Geriatr Psychiatry 2006;21:103-107.

69. Chow TW, Liu CK, Fuh JL, Leung VP, Tai CT, Chen LW, Wang SJ, Chiu HF, Lam LC, Chen QL, Cummings JL. Neuropsychiatric symptoms of Alzheimer's disease differ in Chinese and American patients. Int J Geriatr Psychiatry 2002; 17:22-28.

70. Chong MS, Sahadevan S. Preclinical Alzheimer's disease: diagnosis and prediction of progression. Lancet Neurol 2005;4:576-579.

71. Morris JC. Mild cognitive impairment is early-stage Alzheimer disease: time to revise diagnostic criteria. Arch Neurol 2006;63:15-16.

72. Meguro K, Ishii H, Yamaguchi S, Ishizaki J, Sato M, Hashimoto R, Meguro M, Lee E, Tanaka Y, Kasuya M, Sekita Y. Prevalence and cognitive performances of clinical dementia rating 0.5 and mild cognitive impairment in Japan. The Tajiri project. Alzheimer Dis Assoc Disord 2004;18:3-10.

73. Li M, Ng TP, Kua EH, Ko SM. Brief informant screening test for mild cognitive impairment and early Alzheimer's disease. Dement Geriatr Cogn Disord 2006;21:392-402.

74. Hashimoto R, Meguro K, Yamaguchi S, Ishizaki J, Ishii H, Meguro M, Sekita Y. Executive dysfunction can explain word-list learning disability in very mild Alzheimer's disease: the Tajiri project. Psychiatry Clin Neurosci 2004;58:54-60.

75. Lam LC, Ho P, Lui VW, Tam CW. Reduced semantic fluency as an additional screening tool for subjects with questionable dementia. Dement Geriatr Cogn Disord 2006;22:159-164.

76. Wang PN, Liu HC, Lin KN. The MCI study in Taiwan. Acta Neurol Taiwan 2006;15:66-68.

77. Meguro K, Meguro M, Caramelli P, Ishizaki J, Ambo H, Chubaci RY, Hamada GS, Nitrini R, Yamadori A. Elderly Japanese emigrants to Brazil before World War II: II. Prevalence of senile dementia. Int J Geriatr Psychiatry 2001;16:775-779.

78. Suh GH, Yeon Jung H, Uk Lee C, Hoon Oh B, Nam Bae J, Jung HY, Ju YS, Kil Yeon B, Park J, Hong I, Choi S, Ho Lee J; Korean Galantamine Study Group. A prospective, double-blind, community-controlled comparison of three doses of galantamine in the treatment of mild to moderate Alzheimer's disease in a Korean population. Clin Ther 2004;26:1608-1618.

79. Suh GH, Son HG, Ju YS, Jcho KH, Yeon BK, Shin YM, Kee BS, Choi SK. A randomized, double-blind, crossover comparison of risperidone and haloperidol in Korean dementia patients with behavioral disturbances. Am J Geriatr Psychiatry 2004;12:509-516.

80. Wong WJ, Liu HC, Fuh JL, Wang SJ, Hsu LC, Wang PN, Sheng WY. A double-blind, placebo-controlled study of tacrine in Chinese patients with Alzheimer's disease. Dement Geriatr Cogn Disord 1999;10:289-294.

81. Patterson TL, Semple SJ, Shaw WS, Yu E, He Y, Zhang MY, $\mathrm{Wu}$ W, Grant I. The cultural context of caregiving: a comparison of Alzheimer's caregivers in Shanghai, China and San Diego, California. Psychol Med 1998;28:1071-1084.

82. Cacchione PZ, Powlishta KK, Grant EA, Buckles VD, Morris JC. Accuracy of collateral source reports in very mild to mild dementia of the Alzheimer type. J Am Geriatr Soc 2003;51:819-823.
83. Ross GW, Abbott RD, Petrovitch H, Masaki KH, Murdaugh C, Trockman C, Curb JD, White LR. Frequency and characteristics of silent dementia among elderly Japanese-American men. The Honolulu-Asia Aging Study. JAMA 1997;277:800-805.

84. Mok VC, Wong A, Yim P, Fu M, Lam WW, Hui AC, Yau C, Wong KS. The validity and reliability of chinese frontal assessment battery in evaluating executive dysfunction among Chinese patients with small subcortical infarct. Alzheimer Dis Assoc Disord 2004;18:68-74.

85. Galvin JE, Roe CM, Powlishta KK, Coats MA, Muich SJ, Grant E, Miller JP, Storandt M, Morris JC. The AD8: a brief informant interview to detect dementia. Neurology 2005;65:559-564.

86. Mackinnon A, Khalilian A, Jorm AF, Korten AE, Christensen $\mathrm{H}$, Mulligan R. Improving screening accuracy for dementia in a community sample by augmenting cognitive testing with informant report. J Clin Epidemiol 2003;56:358-366.

\section{AUTHOR AFFILIATIONS}

Wee Shiong Lim MRCP, MMed

Department of Geriatric Medicine

Tan Tock Seng Hospital, Singapore

Mei Sian Chong MRCP

Department of Geriatric Medicine

Tan Tock Seng Hospital, Singapore

Suresh Sahadevan FRCP

Department of General Medicine

Tan Tock Seng Hospital, Singapore 